\title{
April 2014 Imaging Case of the Month
}

\author{
Eric A. Jensen, MD \\ Michael B. Gotway, MD \\ Department of Radiology \\ Mayo Clinic Arizona \\ Scottsdale, AZ
}

Clinical History: A 34-year-old woman presented with complaints of abdominal fullness. The patient described a history of several pneumonias, successfully treated with antibiotics. Liver function testing showed a mild transaminitis. Frontal chest radiography (Figure 1) was performed.

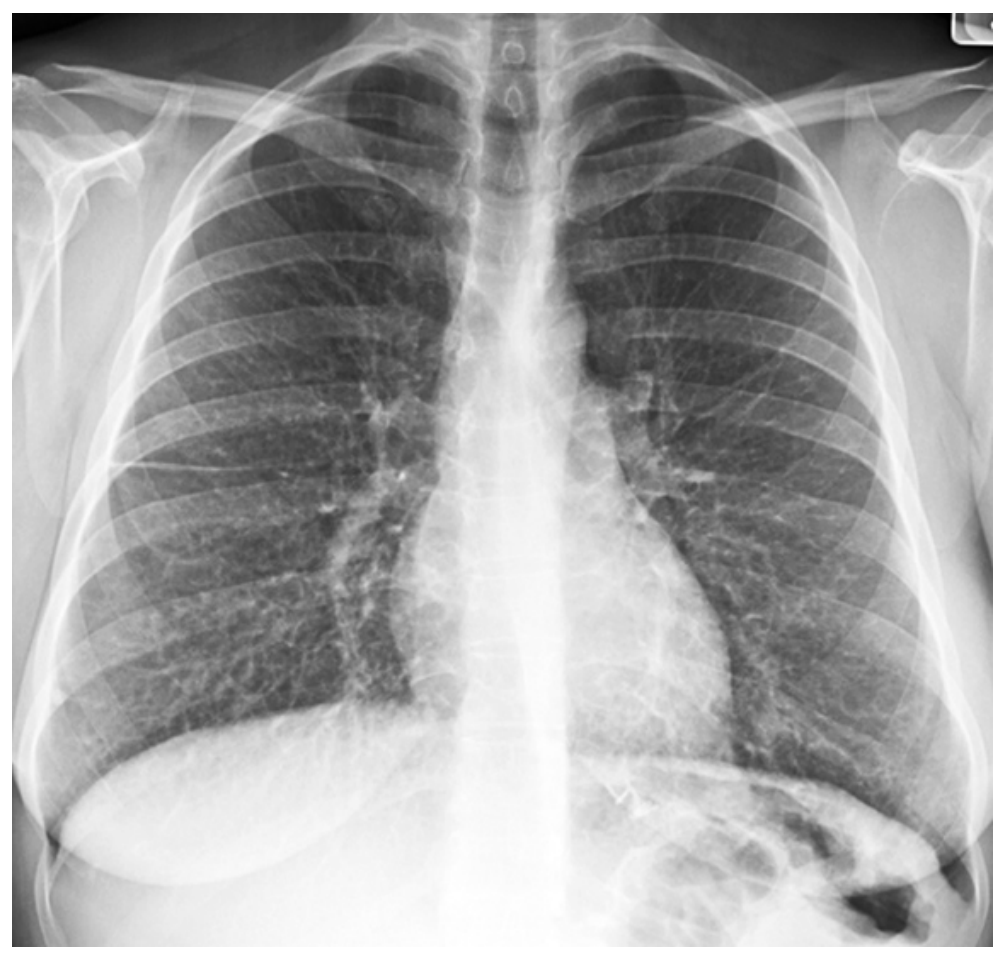

Figure 1. Frontal chest radiograph.

Which of the following statements regarding the chest radiograph is most accurate?

1. The chest radiograph shows multifocal coarse linear opacities suggesting bronchiectasis

2. The chest radiograph shows multifocal ground-glass opacity and consolidation

3. The chest radiograph shows multifocal, somewhat basal predominant linear opacities within diminished lung volumes, suggesting fibrosis

4. The chest radiograph shows multiple small nodules, suggesting a miliary pattern

5. The chest radiograph shows symmetric, basal predominant fine linear and reticular abnormalities without architectural distortion 


\section{Correct!}

5. The chest radiograph shows symmetric, basal predominant fine linear and reticular abnormalities without architectural distortion

The frontal chest radiograph shows normal lung volumes. A fine linear and reticular pattern is present in a symmetric fashion affecting the mid and lower lungs bilaterally (Figure 2).

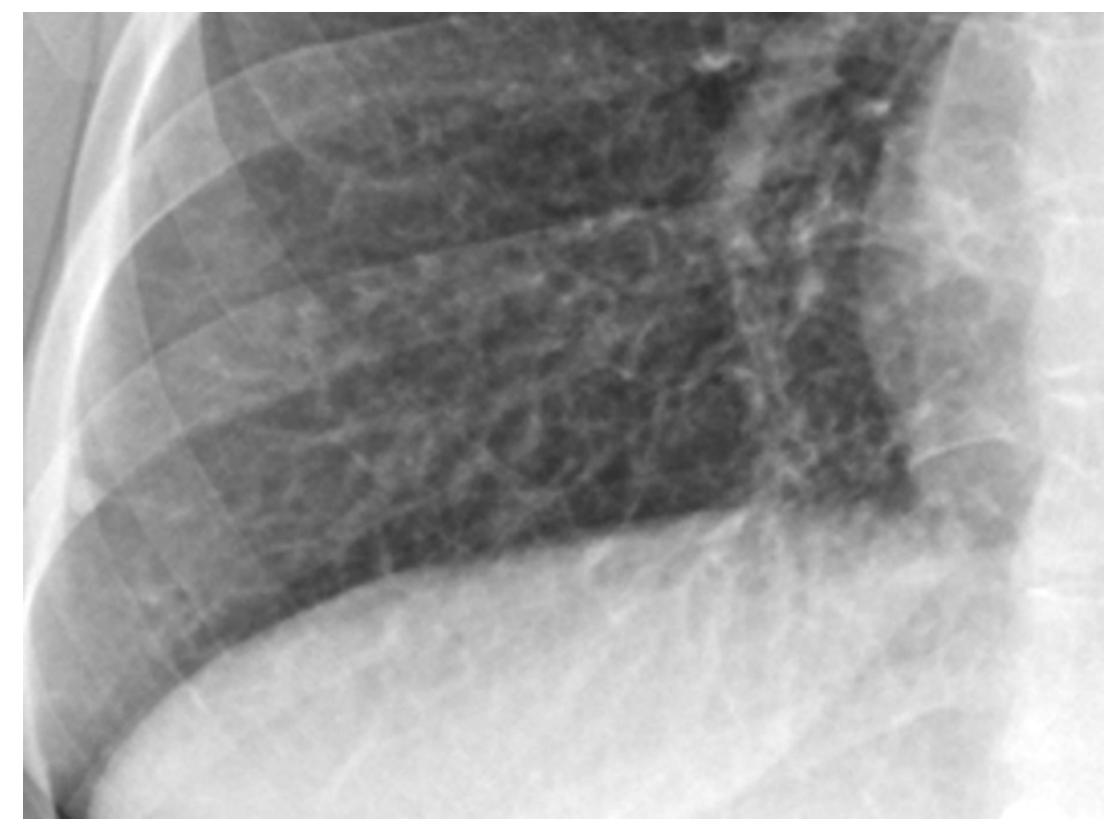

Figure 2. Focused detail image of the right lower lobe shows the radiographic abnormalities to advantage

No pleural effusion or lymphadenopathy is present and the mediastinal contours appear normal. While there are basal linear opacities, there are no features of fibrosis, such as architectural distortion, coarse linear and reticular opacities, traction bronchiectasis, and volume loss. While a small, calcified right base subpleural nodule is present, there is no evidence of numerous, bilateral, small nodules to suggest a miliary pattern. No evidence of ground-glass opacity or consolidation is seen. Basal predominant linear opacities are evident, but their appearance is not suggestive of bronchiectasis- no "tram-tracks" (dilated, thick-walled bronchi seen in longitudinal section) or cystic "ring shadows" (dilated, thick-walled bronchi seen in cross section) are evident.

Which of the following is the least appropriate consideration among the differential diagnostic possibilities for the appearance of the patient's chest radiograph?

1. Atypical pneumonia

2. Cystic fibrosis

3. Hypersensitivity pneumonitis

4. Nonspecific interstitial pneumonitis

5. Pulmonary Kaposi sarcoma 


\section{Correct! \\ 2. Cystic fibrosis}

The linear and reticular abnormalities noted on the chest radiograph could be produced by any of the above disorders, but, among these choices, the basal predominant findings on the radiograph is atypical for cystic fibrosis. Cystic fibrosis may produce linear abnormalities- reflecting bronchial wall thickening and bronchiectasis- but these abnormalities are typically upper lobe predominant, often preferentially affecting the right upper lobe. Furthermore, the lung volumes appear normal, and cystic fibrosis would be expected to produce an obstructive pattern, manifesting on the chest radiograph as increased lung volumes. The other lesions listed may present with normal or nearly-normal chest radiographic findings; when findings are evident, however, they are often most readily visible in the mid and lower lungs.

Additional laboratory data showed hypercholesterolemia with elevated low density lipoprotein-C and reduced levels of high density lipoprotein-C. Pulmonary function testing suggested the presence of restrictive ventilatory defect. The patient underwent thoracic CT (Figure 3) for further characterization of the chest radiographic and pulmonary function testing findings.

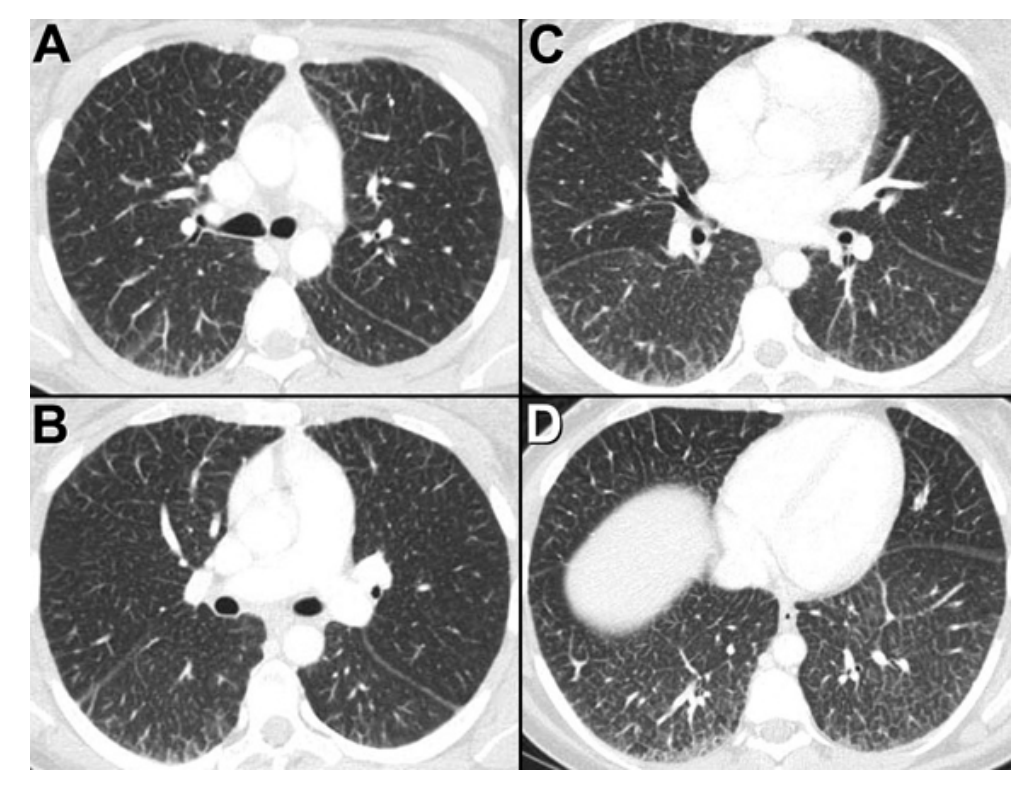

Figure 3. Representative thoracic CT images displayed in lung windows

Which of the following statements regarding this CT examination is most accurate?

1. The thoracic CT shows a diffuse fibrotic process

2. The thoracic CT shows extensive smooth interlobular septal thickening

3. The thoracic CT shows features characteristic of smoking-related interstitial lung disease

4. The thoracic CT shows findings suggesting active infection

5. The thoracic CT shows multifocal basal predominant bronchiectasis 


\section{Correct!}

\section{The thoracic CT shows extensive smooth interlobular septal thickening}

The thoracic CT shows extensive, basal predominant, smooth and regular linear opacities reflecting smooth interlobular septal thickening (Figure 4).

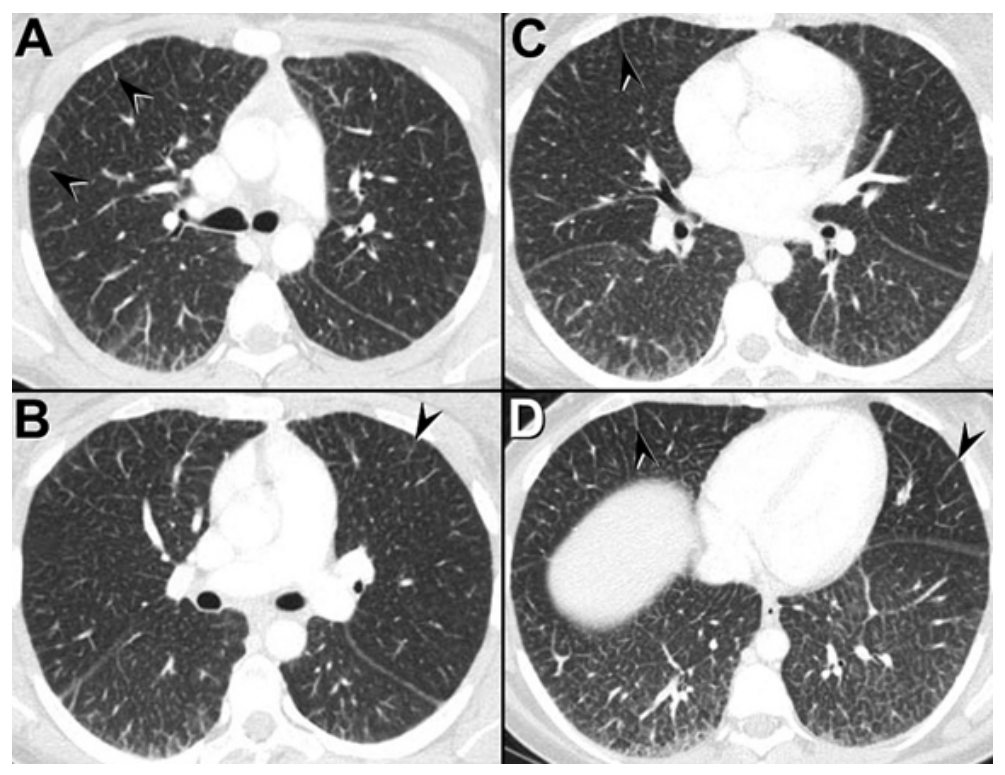

Figure 4. Thoracic CT displayed in lung windows through the mid and lower lungs shows smooth interlobular septal thickening (arrowheads), becoming particularly pronounced in the bases bilaterally.

The central and peripheral airways appear normal- there is no evidence of bronchiectasis. No features of fibrotic lung disease, such as traction bronchiectasis, lobular distortion, honeycomb lung, architectural distortion, and coarse reticulation, are evident. Smoking-related pulmonary diseases may present in variable fashion on thoracic CT, often with features of obstructive lung disease, particularly emphysema, superimposed. Respiratory bronchiolitis typical manifests as upper lobe, small, centrilobular nodules in an asymptomatic patient. Respiratory bronchiolitis-interstitial lung disease occurs in symptomatic, usually heavy smokers, typically shows findings such as bronchial wall thickening, multifocal ground-glass opacity, upper lobe predominant ground-glass opacity centrilobular nodules, and air trapping at thoracic CT. Desquamative interstitial pneumonia usually presents as multifocal, sometimes peripherally predominant, ground-glass opacity, occasionally associated with cystic foci. Langerhans cell histiocytosis usually presents as upper lobe predominant nodules that eventually undergo cystic change and cavitation, eventually resulting in thin-walled cysts resembling severe, confluent, centrilobular emphysema. Combined pulmonary fibrosis and emphysema presents with upper lobe emphysema and lower lobe fibrotic findings. An emerging literature detailing other patterns of smoking-related interstitial abnormalities, variously referred to as respiratory bronchiolitis with fibrosis, air space enlargement with fibrosis, and smoking-related interstitial fibrosis, may manifest as 
upper lobe predominant emphysema associated with interstitial thickening and reticulation, sometimes simulating a cystic appearance, or as upper lobe predominant emphysema with lower lung ground-glass opacity; none of these presentations are evident in this patient. Thoracic CT features of active pulmonary infection various include focal, multifocal, or diffuse ground-glass opacity and / or consolidation, bronchial wall thickening, and small nodules, occasionally with branching configurations- no such abnormalities are seen in this patient).

A more focused history and physical examination was performed and additional laboratory evaluation was pursued. Hepatosplenomegaly was detected, and it was noted that the patient was thrombocytopenic.

What it the most likely relevant historical point discovered as a result of the focused history?

1. Relatives with a history of an inherited metabolic disorder

2. The patient has had a stem cell transplant

3. The patient is a long-time heavy smoker

4. The patient owns two parakeets

5. The patient works as a sandblaster 


\section{Correct! \\ 1. Relatives with a history of an inherited metabolic disorder}

The hepatosplenomegaly and thrombocytopenia suggest a disorder infiltrating the bone marrow. These findings, in addition to the interlobular septal thickening, raise the possibility of a disorder that results in accumulation of abnormal tissue or cells in various organs, producing dysfunction of the affected organs. There are numerous such disorders, but many of them are heritable, and thus a history of family members with similar abnormalities would be strong evidence of a heritable metabolic disorder. The CT findings do not suggest hypersensitivity pneumonitis, nor would this diagnosis explain the historical and physical examination findings; therefore, choice " 4 " is not correct. The history of sandblasting should suggest the possibility of an occupational disorder, specifically pulmonary alveolar proteinosis. While the thoracic CT findings do not show a "crazy paving" pattern, the extensive, smooth interlobular septal thickening is not inconsistent with that consideration. However, the history and physical examination findings could not be explained by pulmonary alveolar proteinosis. As discussed previously, the CT findings, not to mention the history and physical examination findings, cannot be accounted for on the basis of smoking-related disorders. Finally, the history of a stem cell transplant would suggest the possibility of recurrent disease (often malignancy) that prompted transplant, disorders complicating the immunosuppression regimens used for stem cell transplant patients (particularly pulmonary infection), and disorders related to the nature of the transplant process (such as bronchiolitis obliterans or organizing pneumonia as manifestations of graft-versushost disease). As noted above, thoracic CT features of pulmonary infection are not present in this case. Bronchiolitis obliterans and organizing pneumonia usually manifest on thoracic CT as bronchial wall thickening, bronchiectasis, and air trapping, and peripheral and peribronchial consolidation, respectively; these findings are not present in this case.

Further history did disclose other family members with a disorder similar to the patient's complaints, although a clear diagnosis was not established.

Which of the following should be performed to establish the diagnosis for this patient?

1. ${ }^{68} \mathrm{Ga}$ citrate scanning

2. Bone marrow aspiration

3. Bronchoscopy with bronchoalveolar lavage and transbronchial biopsy

4. Percutaneous transthoracic needle biopsy

5. Surgical lung biopsy 


\section{Correct! \\ 2. Bone marrow aspiration}

Given the interlobular septal thickening, bronchoscopy with bronchoalveolar lavage and transbronchial biopsy could obtain diagnostic tissue, assuming the thickened septae contain abnormalities that would allow a specific diagnosis. However, as noted previously, the historical, physical examination, and laboratory features suggest a process infiltrating the bone marrow, and therefore examination of the bone marrow may have a higher likelihood of providing the actual diagnosis. Much like bronchoscopy with bronchoalveolar lavage and transbronchial biopsy, surgical lung biopsy would be capable of determining the cause of the interlobular septal infiltration, but is needlessly invasive when bone marrow aspiration may provide similar, or even superior, information. ${ }^{68} \mathrm{Ga}$ citrate scanning would not provide diagnostically useful or management-altering information in this case. Pulmonary tracer at ${ }^{68} \mathrm{Ga}$ citrate scanning uptake could represent either active pulmonary inflammation or neoplastic infiltration, but a pulmonary abnormality is already known; in contrast, the absence of tracer uptake within the lung parenchyma would not alleviate the need to establish a tissue diagnosis. Percutaneous transthoracic needle biopsy is typically employed for focal pulmonary abnormalities, not multifocal or diffuse interstitial abnormalities; the latter are difficult to target with and typically not amenable to percutaneous techniques.

Bone marrow aspiration showed the presence of lipid-laden, foamy cells (Niemann-Pick cells). Because these cells, while characteristic of Niemann-Pick disease, are not diagnostic of that disorder (they can also be seen in cholesterol ester storage disease, Wolman disease, lipoprotein lipase deficiency, and, occasionally, $\mathrm{G}_{\mathrm{M} 1}$ gangliosidosis type 2), further measurement of acid sphingomyelinase activity in peripheral white blood cells was performed and was found to be decreased to $<10 \%$ normal activity. Genetic analysis showed the deltaR608 mutation affecting the SMPD1 gene, located on bands 11p15.1-p15.4, which normally codes for the lysosomal enzyme acid sphingomyelinase. These findings established the diagnosis of Niemann-Pick disease, type B.

Diagnosis: Niemann-Pick disease, type B.

\section{Discussion}

Niemann-Pick disease (NPD) is a rare, autosomal recessive lysosomal storage disorder due to a deficiency of acid sphingomyelinase, which results in the accumulation of sphingomyelin within the monocyte-macrophage system. Sphingomyelinase is a lysosomal enzyme encoded by the SMPD1 gene located on bands 11p15.1-p15.4. This accumulation produces a foam cell- the Pick cell- in affected tissues, which is the characteristic histopathologic finding of this disorder.

NPD is often divided into two distinct subtypes based on phenotype- NPD-A and NPDB. An "NPD-C" is also described, but is a genetically distinct abnormality that results from defective intracellular handling of cholesterol, resulting in a secondary accumulation of glycosphingolipids. 
NPD-A often affects patients of Ashkenazi Jewish decent, although NPD may affect any race, and usually manifests in infancy as a severe neurodegenerative disorder characterized by failure to thrive, psychomotor deficiency, cherry-red macula, hepatosplenomegaly, with death usually resulting by the age of 3 years.

NPD-B may affect persons of any race, but often is seen in patients from North Africa, Saudi Arabia, and Turkey. NPD-B presents with a spectrum of severity related to the amount of residual acid sphingomyelinase activity, and is characterized by hepatosplenomegaly, thrombocytopenia, dyslipidemia, and interstitial lung disease; neurological involvement is usually absent or minimal. Pulmonary involvement in NPD-B is common and results from the interstitial accumulation of lipid-laden macrophages, notably within the alveolar septae, bronchial walls, and pleura. This accumulation results in a restrictive ventilatory abnormality which may range from practically asymptomatic to respiratory failure. On chest radiography, the interstitial infiltration produces a linear or reticular pattern that reflects the presence of thickened interlobular septae, sometimes accompanied by vague areas of increased attenuation and small, possibly calcified lung nodules. At thoracic $\mathrm{CT}$, these abnormalities may produce a "crazy paving" pattern." The interstitial abnormalities are typically more pronounced in the bases. Additional imaging findings in patients with NPD-B include coronary arterial calcification, reflecting premature coronary arterial atherosclerosis (which may be a notable finding given the relatively young age of affected patients), hepatosplenomegaly, osteopenia / osteoporosis, delayed skeletal maturation, and adrenal lesions. Recurrent pneumonias and cirrhosis with features of portal hypertension have been described.

The diagnosis of NPD is usually made by demonstrating reduced acid sphingomyelinase activity within peripheral leukocytes of cultured fibroblasts, or through the detection of characteristic mutations in the acid sphingomyelinase gene. Bone marrow aspiration may demonstrate the characteristic, lipid-laden foamy Niemann-Pick cell.

\section{References}

1. Simpson WL Jr, Mendelson D, Wasserstein MP, McGovern MM. Imaging manifestations of Niemann-Pick disease type B. Am J Roentgenol AJR 2010; 194(1):W12-19. [CrossRef] [PubMed]

2. Muntaner I, Galmés A, Chabás A, Herrera M. Imaging features of type-B NiemannPick disease. Eur Radiol 1997; 7:361-364. [CrossRef] [PubMed] 